\title{
LA ESPECIALIDAD MEDICA DE GESTIÓN EN SALUD EN LA UNIVERSIDAD NACIONAL MAYOR DE SAN MARCOS: 30 AÑOS FORMANDO GESTORES PARA EL SECTOR SALUD
}

\author{
German Felipe Rivera Del Río ${ }^{1, a}$, Edson Valdivia Veraª, ${ }^{2, a}$ Alida Esther Palacios Enriquez ${ }^{3, a}$, Javier Del Campo Sánchezª, \\ Luis Felipe Rojas Cama ${ }^{1, b}$, Nadia Mariela Lingán Cubas ${ }^{1, c}$, Isabel Carolina Tello Rivera ${ }^{1, c}$, \\ José Carlos Del Carmen Sara ${ }^{4, d}$
}

\begin{abstract}
RESUMEN
Se presenta un recuento histórico de los 30 años de la especialidad médica de Gestión en Salud en la Universidad Nacional Mayor de San Marcos. La especialidad fue creada en 1988 como Medicina General Integral en respuesta a la escasez de médicos especialistas en gestión. El nombre de la especialidad se mantuvo hasta 1994, cuando cambia a Medicina Integral y Gestión en Salud para enfatizar su orientación gerencial. En 2002, se modifica el plan curricular considerando la importancia de tener una especialización médica exclusiva de gestión para el sector salud. La especialidad es pionera en una formación gradual en tres niveles: microgestión, mesogestión y macrogestión. El nuevo plan curricular permitió a los residentes la posibilidad de mejores rotaciones en instituciones públicas y privadas. A partir del 2007, la especialidad es denominada Gestión en Salud, cuyos médicos especialistas mantienen su sólida formación para desempeñarse en los procesos de decisión, conducción, dirección y operatividad de los sistemas de salud. La visión de los creadores de la especialidad se encuentra vigente, los especialistas de Gestión en Salud se desempeñan en las diferentes instituciones del sistema de salud, aportando sus conocimientos y habilidades, y generando un impacto en la salud de la población peruana.
\end{abstract}

Palabras clave: Gestión en salud; Administración de los servicios de salud; Especialización; Perú (fuente: DeCS BIREME).

\section{THE MEDICAL MAJOR OF HEALTH MANAGEMENT IN UNIVERSIDAD NACIONAL MAYOR DE SAN MARCOS: 30 YEARS TRAINING MANAGERS FOR THE HEALTH SECTOR}

\begin{abstract}
A historical account of the 30 years of the medical major of Health Management at Universidad Nacional Mayor de San Marcos is presented. The major was created in 1988 as Comprehensive General Medicine in response to the shortage of management specialists. The name of the major remained until 1994, when it changed to Integral Medicine and Health Management to emphasize its managerial orientation. In 2002, the curricular plan was modified considering the importance of having an exclusive medical specialization in management for the health sector. The specialty is a pioneer in a gradual education in three levels: micro-management, meso-management, and macro-management. The new curricular plan allowed residents the possibility to access better rotations at public and private institutions. Since 2007, the major is labeled Health Management, and its specialists maintain their solid training that enables them to perform in the decision-making, management, administration, and operation processes of the health systems. The vision of the creators of this major is current to this day; Health Management specialists work in the different institutions of the health system, contributing their knowledge and skills, and generating an impact on the health of the Peruvian population.
\end{abstract}

Keywords: Health management; Administration of health services; Major; Peru (source: MeSH NLM).

\section{INTRODUCCIÓN}

La especialidad de Gestión en Salud ha cumplido 30 años desde su creación en la Universidad Nacional Mayor de San Marcos (UNMSM). En su inicio, se creó para dotar de médicos especialistas con visión integral en el cumplimiento de su rol asistencial en la comunidad. Sin embargo, al transcurrir los años, los propios especialistas

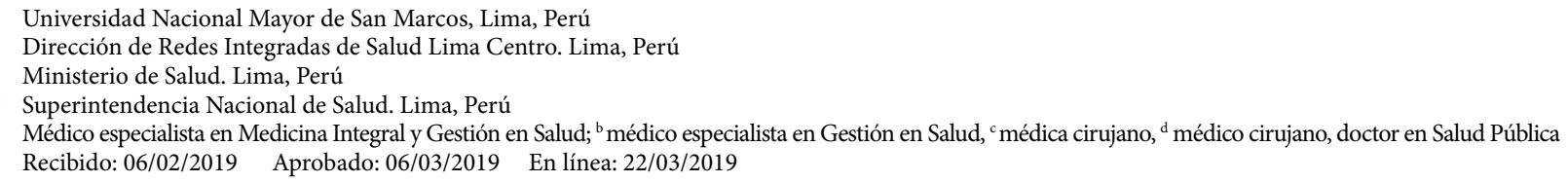


vieron la necesidad de un entrenamiento más específico en el campo de la gestión, por lo que, hoy en día la especialidad provee competencias dirigidas a desarrollar la gestión en diversos niveles del sistema de salud.

La especialidad fue concebida en base a las experiencias obtenidas de la Medicina Familiar de España y del modelo educativo cubano a fines de los años 70 y 80 , respectivamente. Al igual que España, Cuba inicia la especialización tomando en consideración aspectos sociales, económicos y culturales de la población; a fines de los ochenta Cuba creó la especialidad de Organización y Administración de Salud Pública, con un enfoque y preparación gerencial de los residentes. Sin embargo, pese a los buenos intentos por mantener la especialidad, Cuba dejó de formar especialistas, en este rubro, en $1990^{(1,2)}$

Lo sucedido en Cuba, conlleva a una reflexión acerca de la especialidad de Gestión en Salud en nuestro país, su ausencia podría desencadenar un vacío importante de aporte científico-técnico necesario para el sistema de salud. Es necesario, destacar la importancia de la transformación del médico asistencial que se especializa en Gestión en Salud; profesionales altamente capacitados, con habilidad y sólidos conocimientos capaces de proyectar las acciones de salud a la población. Consideramos que la continuidad y el desarrollo de la especialidad no se dio en los profesionales de Cuba, como si sucedió en Perú.

El especialista de Gestión en Salud tiene como base su formación teórica-práctica del proceso salud-enfermedad; además de conocimientos en administración, gerencia y gestión de la salud pública y de los diversos factores determinantes que afectan la salud, tanto económicos, culturales, psicológicos y biológicos. Además, debe fortalecer sus principios políticos, humanistas, éticos y ampliar sus conocimientos científicos con el objetivo de identificar problemáticas, analizar sus causas, plantear posibles respuestas e implementar soluciones a corto, mediano y largo plazo. Este modelo fue tomado de la desaparecida especialidad de Organización y Administración de Cuba.

Son escasos los países que tienen una especialidad con el perfil del médico especialista de Gestión en Salud peruano. En España, la especialidad de Medicina Preventiva y Salud Pública incluye en su perfil aspectos de gestión, planificación y evaluación de los servicios de salud, sumado a la prevención e identificación de daños en la población. En Chile, la especialidad de Salud Pública, incluye la Gestión Sanitaria, Epidemiología, Promoción de la Salud, Estadística, así como, obtener destrezas para ejercer roles de liderazgo en investigación. En México, existe una especialidad denominada Calidad en la Atención Clínica; y en Uruguay, existe la especialidad de Administración de Servicios de salud ${ }^{(3,4)}$.
La especialidad médica de Gestión en Salud de Perú se diferencia del resto por tener tópicos meramente teóricoprácticos de la gerencia en el sector salud. En otros países, la gestión en el sector salud se incluye como parte de las rotaciones, cursos o tópicos específicos, no siendo la raíz principal de la especialidad. La especialidad de Medicina Preventiva y Salud Pública de España es considerada básicamente como no hospitalaria; además, es apoyada por el programa de maestría de Salud Pública durante el primer año de formación. Esta situación también es compartida por la especialidad de Medicina Familiar y Comunitaria, y la de Medicina del Trabajo. En Perú, el inicio de la especialidad es muy parecida a la que actualmente se desarrolla en España ${ }^{(3)}$.

En un estudio realizado el 2011 por la Organización Panamericana de la Salud, se encontró que, en 14 países de América Latina, el $5 \%$ de las especialidades médicas ofrecidas, tenían entrenamiento en planificación y organización, siendo lo mayoritario las especialidades clínicas y quirúrgicas en cerca del $50 \%{ }^{(5)}$. En Perú, la UNMSM es la única universidad que ofrece la especialidad con el nombre de Gestión en Salud como residentado exclusivo para médicos. En otras universidades tiene la denominación de Administración en Salud o Administración y Gestión en salud. En forma general, la formación del médico especialista de Gestión en Salud tiene que ver con los procesos de gestión (planificación, organización, dirección y control), diseñar programas de intervención sanitaria, ejecutar y evaluar proyectos de inversión, coordinar políticas nacionales de salud, mejoramiento de los servicios de salud, auditoria en salud, calidad en salud, investigación y docencia e investigación operativa en Epidemiología.

En los diferentes lugares y áreas donde se desempeña el médico especialista de Gestión en Salud ejerce un poder de cambio y mejora constante, el cual es identificado rápidamente por los jefes, directores o gerentes. El Dr. Urquizo menciona cómo ha visto crecer la especialidad en el Hospital San Bartolomé, específicamente en la Oficina de Planeamiento, donde se pasó del sentido común a la planificación, análisis y evaluación periódica, produciendo una mejora de los procesos hospitalarios con eficiencia y calidad (entrevista al Dr. Urquizo, Decano del Consejo Regional III Lima del Colegio Médico del Perú).

El objetivo del presente artículo es presentar un recuento histórico de los 30 años trascurridos desde la creación de la especialidad médica de Gestión en Salud en la Universidad Nacional Mayor de San Marcos, desde 1988 hasta la actualidad.

\section{ANTECEDENTES HISTÓRICOS DE LA ESPECIALIDAD}

Entre 1985 y 1990 se empieza a forjar los primeros esbozos de lo que sería la especialidad, y fue durante el periodo 
del Ministro de Salud, Dr. David Tejada Rivero donde se vio la necesidad de un sistema de salud moderno y eficaz. Por tanto, una de sus primeras medidas fue ejecutar la descentralización de la salud y que este se lleve a cabo por profesionales idóneos y líderes en salud. Al crearse las unidades departamentales de salud (instituciones coordinadoras entre hospitales, centros de salud, comedores populares, etc.) se generó la necesidad de que estos profesionales se encarguen y dirijan estas instituciones. La Dra. Maria Fe Malaspina, refiere que, a solicitud del Ministro de Salud, se vio la necesidad de proporcionar recursos humanos para la reforma en salud que se estaba forjando y juntamente con el Dr. Contreras, elaboraron una propuesta (entrevista realizada a la presidenta del Comité de 1998 a 2007 de la especialidad de Gestión en Salud).

Una problemática de esos años era que los profesionales de la salud tenían poca experiencia en las áreas requeridas por la reforma. Si bien, la escuela de Salud Pública había brindado cursos de capacitación en temas afines, estos no correspondían con la realidad que se necesitaba en esos años. Recuerda la Dra. Malaspina que, ante el ministro y autoridades en una reunión nacional mencionó: «...debo reconocer que, al momento, la evaluación realizada, muestra que no se cuenta con profesionales competentes para la conducción de las unidades departamentales del país; tengo que decir, como docente universitario, que la formación que se da en todas las universidades del país es netamente asistencialista, muy de espaldas a la realidad de la salud del país...». (entrevista realizada a la presidenta del Comité de 1998 a 2007 de la especialidad de Gestión en Salud).

\section{CREACIÓN DE LA ESPECIALIDAD Y SUS PRIMEROS AÑOS (1988-2007)}

Ante esta problemática, la UNMSM propone la creación de un programa especial de entrenamiento y formación de médicos especialistas. El Dr. Francisco Sánchez Moreno, decano de la Facultad de Medicina de ese periodo, refiere que la propuesta inicial coincidía con otros modelos implementados en sistemas de salud de países europeos y en Latinoamérica tenía muchas coincidencias con el modelo cubano, con un perfil de especialista que pueda realizar atención en el primer nivel y que, a su vez pueda contribuir a su gerenciamiento. Es así como surge la especialidad de Medicina General Integral a fines de 1988.

El Dr. Sánchez Moreno refiere: «...en 1989 la creación de la especialidad recibió el apoyo del Ministro de Salud Dr. Pinillos, quien ante el requerimiento de vacantes para esta nueva especialidad por parte de la UNMSM, convocó a todas las universidades, otorgando 40 vacantes a la UNMSM, 20 a la Universidad Peruana Cayetano Heredia, 20 a la Universidad Federico Villarreal y 10 a la Universidad Nacional San Luis Gonzaga» (entrevista realizada al Dr. Francisco Sánchez Moreno exdecano de la facultad de medicina de la UNMSM).
La Dra. Malaspina refiere: «...el comienzo fue difícil, se hecho andar a la especialidad con 24 ingresantes, con cuatro rotaciones asistenciales y una administrativa; la especialidad necesitaba un enfoque más gerencial para poder satisfacer a los funcionarios en la toma de decisiones...... La especialidad durante los siguientes años adoleció de problemas en relación con las vacantes (que disminuyen por pasar a plazas asistenciales) y rotaciones.

En 1995, siendo presidente el Dr. Víctor Orihuela, la especialidad cambia su nombre a Medicina integral y Gestión en Salud, con el fin de enfatizar su orientación gerencial. El departamento de Medicina Preventiva y Salud Pública de la facultad de Medicina de la UNMSM asumió el dictado de las clases teóricas, sin embargo, era evidente que se necesitaban más conocimientos y habilidades gerenciales. Otro problema fue la variabilidad en el número de plazas que se ofrecían cada año; generalmente ocasionado por atender prioridades o directivas del Ministerio de Salud para otras especialidades.

A partir del aporte de las promociones de 1998 y 1999, y de muchos egresados de Medicina Integral y Gestión en Salud surgen las primeras iniciativas para que la especialidad adopte el nombre de Gestión en Salud. Para 1999, los ingresantes a la especialidad realizaban rotaciones en gestión y cursos teóricos. En 2002 se formalizó un nuevo plan curricular, aprobado por el vicerrectorado académico, con contenido íntegramente dedicado a la gestión sanitaria. Este cambio originó la creación de otra especialidad en la facultad de Medicina de la UNMSM, la de Medicina Familiar. La evolución de la especialidad Gestión en Salud ha tenido hitos importantes los que se pueden apreciar en la Figura 1.

\section{LA ESPECIALIDAD MEDICA DE GESTION EN SALUD A PARTIR DEL 2007}

En 2007, la especialidad cambió su nombre a Gestión en Salud. El nuevo plan curricular expresa un modelo formativo que responde a la demanda social de la especialidad y a la necesidad de una gestión en salud eficiente; está basado en competencias desarrolladas a través de cursos y rotaciones durante los tres años de formación, donde el egresado culmina el programa formativo siendo capaz de usar herramientas y tecnologías que le permitan desempeñar los procesos de decisión, conducción, dirección y operatividad de los sistemas de salud del ámbito nacional, regional y local, tanto en su componente público como privado.

La especialidad médica de gestión en salud de la UNMSM, es la pionera en Perú con un modelo formativo a través de cursos teóricos y rotaciones con gradualidad formativa en los tres niveles de gestión: microgestión, mesogestión y macrogestión. Otras universidades tienen una especialidad similar, pero con nombres diferentes: en la Universidad Peruana Cayetano Heredia, Universidad San Martín 


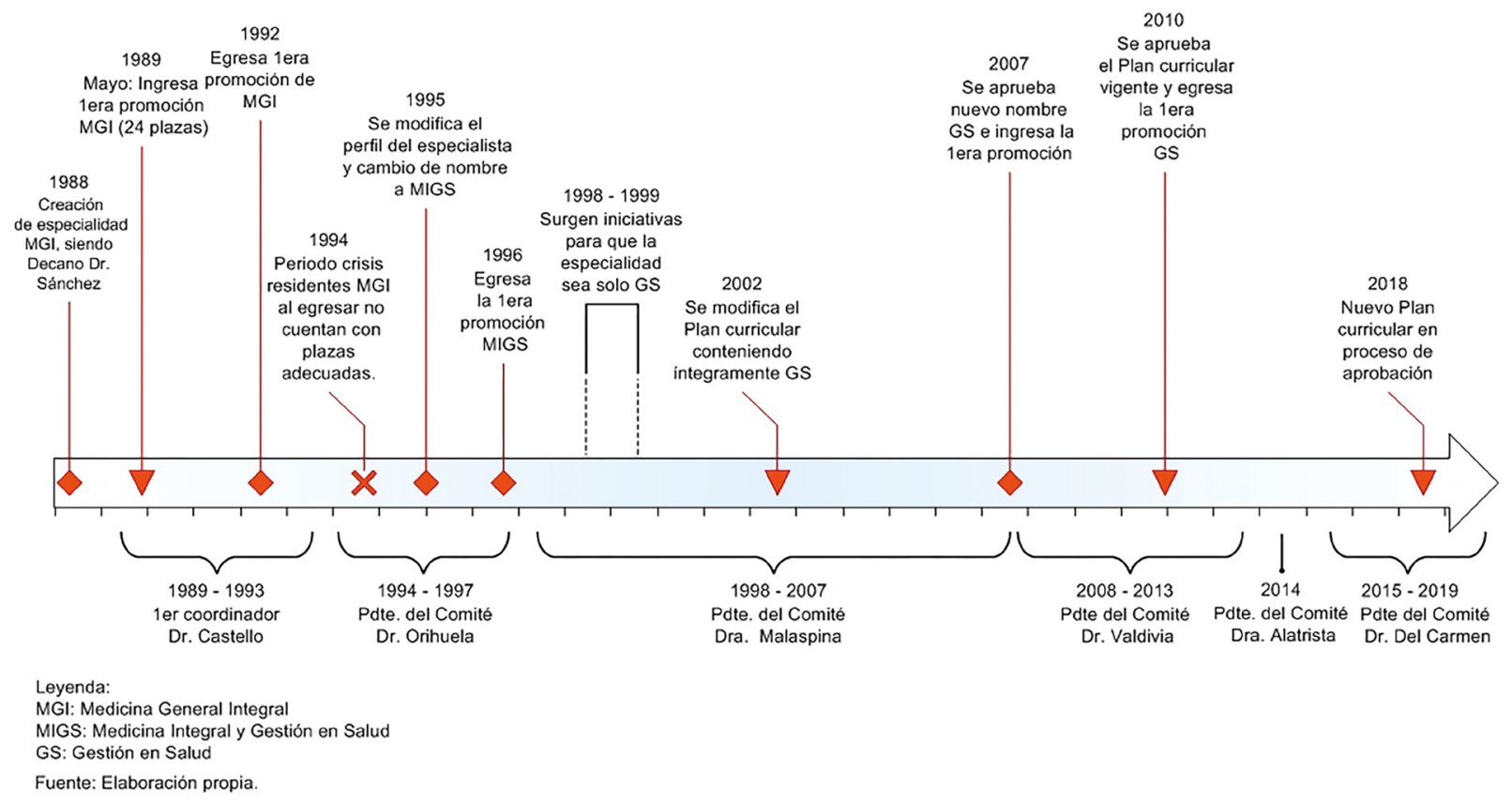

Figura 1. Especialidad médica de Gestión en salud de la Universidad Nacional Mayor de San Marcos: Recuento histórico desde su creación

de Porres y Universidad Ricardo Palma se denomina Administración en Salud; en la Universidad Nacional de San Agustín de Arequipa y Universidad Privada San Juan Bautista se llama Administración y Gestión en Salud; y en la Universidad Privada Antenor Orrego se le denomina Gestión y Administración en Servicios de Salud (6-9).

En América Latina, existen especialidades médicas afines a la especialidad de Gestión en Salud de la UNMSM. En Chile y Argentina la especialidad médica es afín a la especialidad de Salud Pública, enmarcada en el nivel de macrogestión, con formación en los denominados campos de la Salud Pública: Gestión Sanitaria, Epidemiología, Promoción de la Salud, Bioestadística y Salud Ambiental. En Paraguay, la especialidad se llama Salud Pública y Administración Hospitalaria, y se relaciona a nivel de macrogestión y mesogestión. En México se cuenta con la especialidad de Calidad de la Atención Clínica, enfocada más en la microgestión. En Uruguay, la especialidad toma el nombre de Administración de Servicios de Salud y tiene campo de acción en macrogestión, mesogestión y microgestión ${ }^{(10-12)}$.

En España, existe la especialidad de Medicina Preventiva y Salud Pública, que aparece en 1978 con el programa Médico Interno Residente (MIR), en el que se definen cinco campos de acción o áreas profesionales específicas: Epidemiología, Administración Sanitaria, Medicina Preventiva, Salud Ambiental y Laboral y Promoción de la Salud ${ }^{(13)}$. En la Unión Europea, a través de la Directriz 93/16/CEE se estandariza que los países miembros deben cumplir con un programa de residencia de al menos cuatro años para esta especialidad, la misma que, con variantes de nombres, existe en Francia, Irlanda y Reino Unido, Austria, Finlandia, Grecia, Islandia, Italia, Liechtenstein, Noruega, Suecia, entre otros ${ }^{(7)}$. En Estados Unidos, existe el programa de residencia médica de Medicina Preventiva desde hace muchos años, y la gestión siempre ha sido el mayor componente de todas las áreas de especialidad de Medicina preventiva (Medicina Aeroespacial, Medicina Preventiva General y Salud Pública, Medicina Ocupacional), por los que se conformó la subespecialidad de Medicina Gerencial (14).

Hace más de 30 años, una editorial publicada en JAMA afirmó que: «...los médicos-ejecutivos pueden ser los únicos capaces de hacer frente a los cambios rápidos y profundos y complejidades médicas, financieras y éticas que ahora acosan a la práctica de la medicina» y que, «...el cuidado de la salud es un ejemplo perfecto de una industria en la que las decisiones no se pueden tomar sólo con números, pero que los números no se pueden ignorar». Si los médicos pretenden mantener cierto control sobre la industria del cuidado de la salud y garantizar la calidad y la continuidad de la atención, algunos de ellos deben estar capacitados tanto en la gestión como en la medicina. Las decisiones financieras deben tomarse dentro del contexto 
de un buen juicio médico, para que el resultado final no domine totalmente la toma de decisiones ${ }^{(15)}$.

En ese mismo sentido, Ackerly señala que gran parte de la generación actual de médicos-líderes han logrado sus roles actuales de una manera que puede ser descrita como «gestores accidentales», porque los médicos a menudo son identificados y promovido a posiciones de liderazgo basándose en sus logros profesionales y distinción clínica, con menos énfasis en sus habilidades de gestión y experiencia. Desafortunadamente, gran parte de estos médicos líderes en la clínica y en la medicina académica, han asumido posiciones de gestión sin ningún entrenamiento formal, formación o habilidades en planificación, finanzas, marketing, adquisiciones, recursos humanos, o estrategia ${ }^{(16)}$.

Es así, que la UNMSM, ha estado aportando profesionales médicos con sólida formación en gestión durante estos últimos 30 años. De acuerdo a los datos disponibles en el Colegio Médico de Perú, existen registrados 260 médicos especialistas en Medicina General Integral, 99 en Medicina Integral y Gestión en Salud y 54 en Gestión en Salud, que suman un total de 413 , esta cifra es casi el doble de los registrados en especialidades afines de otras universidades: Administración de Hospitales (43), Administración de Salud (172) y Administración y Gestión en Salud (4). Gran parte de los especialistas en Administración en Salud obtuvieron su título por la modalidad de titulación por competencias, en tanto, la UNMSM, en los distintos nombres que tuvo la especialidad en estos 30 años, nunca ha emitido títulos bajo esa modalidad ${ }^{(17)}$.

En el aspecto laboral, los médicos especialistas en Gestión en Salud de la UNMSM se encuentran insertados en entidades e instituciones públicas y privadas, desempeñándose en diversas áreas de la gestión sanitaria. En 2016, el Comité de Posgrado de la Especialidad de Gestión en Salud de la Facultad de Medicina de la UNMSM, realizó un seguimiento a 82 de sus egresados y recogió información sobre su ubicación dentro de la estructura organizacional de las entidades o instituciones en las que laboraban, se encontró que se desempeñaban en diversas áreas como: planeamiento, calidad, gestión de emergencias y desastres, gestión de servicios de salud privados, proyectos de inversión, aseguramiento en salud, cobertura y evaluación de prestaciones, dirección hospitalaria, dirección de direcciones regionales de salud, dirección de red de salud, evaluación de tecnologías en salud, regulación de prestaciones, epidemiología, gestión clínica, financiamiento, presupuesto, gestión de recursos humanos en salud, control interno, evaluación de políticas, evidencias en salud pública, inteligencia sanitaria, supervisión de IPRESS, entre otras.

\section{PERSPECTIVA DE LA ESPECIALIDAD}

La evolución del programa académico de segunda especialización médica en Gestión en Salud durante sus primeros 30 años, refleja la influencia de diversos desafíos, en algunos casos contrapuestos, que marcaron el modelo, concepción y desempeño de nuestro sistema sanitario. Por un lado, la clara identificación en sus inicios, de la atención primaria, la gestión territorial y la participación comunitaria, como un importante medio para promover el cuidado de la salud, y por otro lado, el especial énfasis que en la década del 90 se dio a la búsqueda de eficiencia en el uso de recursos, con un foco en la salud individual, que derivaron en generar estrategias de «autogestión», incorporando mecanismos de recaudación en servicios públicos y la búsqueda de ahorros, que desvirtuaron su esencia en la búsqueda de un mejor gerenciamiento.

El médico especialista de Gestión en Salud debe ampliar su campo de acción: del ámbito técnico (generador de normas, responsable de subprocesos) a un líder en el sector salud y agente de cambio. Eso llevará al especialista a ejercer una efectiva gerencia social, con mirada territorial, que lo constituye en un actor político, orientado a la búsqueda de una mejora en el bienestar de la población. Este rol, resulta particularmente relevante, en un nuevo escenario, en que organizaciones como la OCDE (Organización de Cooperación para el Desarrollo Económico), plantean el logro de indicadores para el desarrollo del país.

El Dr. Luis Robles refiere: «A lo largo del tiempo, como corresponde, las competencias de los colegas que hacen la especialidad se van haciendo más exigentes. En el mundo de hoy, el campo de gestión en salud requiere de mayores competencias y que se han ido profundizando. Los especialistas requieren muchas mayores competencias, entonces, lo que les estamos ofreciendo en la universidad tiene la capacidad de alcanzar esas competencias o como ocurre en la vida real optan por otras fuentes de ir mejorando sus conocimientos» (entrevista realizada al Dr. Luis Robles, docente invitado de la especialidad de Gestión en Salud).

Nuestro sistema de salud es producto de una construcción progresiva, con diferentes protagonistas en tiempos distintos, que han trazado un camino que debemos evitar reinventar en cada cambio de gobierno. Es indispensable la existencia de una masa crítica, que pueda identificar y sostener las mejores estrategias e intervenciones para el cambio; así como, alertar sobre aquellas que pudieran amenazar y deteriorar el sistema, ello constituye un nuevo desafío para nuestros médicos especialistas de Gestión en Salud.

La toma de decisiones para la mejora de la gestión en las instituciones, así como el diseño y evaluación de las 
estrategias e intervenciones en salud, requieren de un análisis de la información sistematizada y pertinente. Los médicos especialistas de Gestión en Salud generan conocimiento y publicaciones que deben ser más difundidos y tomados en cuenta por los decisores en salud. Existe hoy un importante aporte de estos médicos especialistas a las diversas instituciones, públicas y privadas, no solo del aparato prestador, sino también en los administradores de fondos, órganos supervisores, e incluso en las industrias relacionadas al campo de la salud.

Actualmente, en su proceso de especialización, los residentes de gestión en salud realizan rotaciones en instituciones públicas de salud (Ministerio de Salud, Essalud, SUSALUD, SIS, Direcciones Regionales de Salud, Ministerio de Economía y Finanzas, Ministerio de la Mujer y Poblaciones Vulnerables), e instituciones privadas (aseguradoras, áreas de gestión/ dirección de clínicas). Al culminar su especialización, los egresados tienen el potencial de elegir algún campo o rama de la gestión que le resulte afín, lo que facilita su inserción laboral.

La Dra. Nilda Osorio refiere: «los especialistas de Gestión en Salud ejercen poder de cambio en el sector en salud, contribuyen a mejorar la rectoría del Ministerio de Salud, siendo partícipes de una mejor coordinación con los municipios; participan en los procesos de mejora de la atención del usuario, como por ejemplo la simplificación administrativa desarrollando diversas estrategias; reducen los tiempos de espera, en un trabajo en conjunto con la oficina de calidad de los hospitales; mejorando el clima laboral y la satisfacción de los usuarios, siendo parte de la evaluación continua de instituciones de salud, aplicando herramientas de gestión para la mejora» (Entrevista a la Dra. Nilda Osorio egresada de la Especialidad de Medicina General Integral de UNMSM).

Los médicos especialistas de gestión en salud también asumen retos gerenciales, como directores generales y ejecutivos en el Ministerio de Salud o como gerentes en el Seguro Social (EsSalud). Asimismo, han participado en hitos importantes, como la creación de la primera norma de categorización, así como la contribución en la creación de los centros de apoyo para dependientes de sustancias psicoactivas (antes llamadas «comunidades terapéuticas») con el apoyo de la Comisión Nacional para el Desarrollo y Vida sin Drogas (DEVIDA) y CEDRO.

En una encuesta realizada el 2019, por el equipo que elaboró este artículo, a médicos especialistas de Gestión en Salud egresados de la UNMSM (104 encuestados), se encontró que el $84 \%$ se insertaron laboralmente al culminar la residencia, y se establecieron en los diferentes niveles de gestión sanitaria: macrogestión (38\%), mesogestión (34\%) y microgestión (27\%). Además, se encuentran en el sector salud desempeñando funciones de: dirección (47\%), supervisión-control (21\%), planificación (18\%) y organización (14\%). Siendo principalmente las instituciones públicas donde estos especialistas laboran (80\%) frente a instituciones privadas (20\%). Estos especialistas, actualmente se encuentran contratados (42\%), designados en cargos de confianza (20\%), nombrados $(26 \%)$ y trabajando como consultores independientes (12\%). El $20 \%$ de los encuestados ha realizado investigación que se encuentra publicada en revistas indizadas.

Estos especialistas han publicado investigaciones sobre temas relacionados a la reforma del sector salud en Perú, el presupuesto por resultados, asociaciones público-privadas hasta temas de inversión en salud ${ }^{(18-21)}$. La optimización de los procesos de planeamiento, organización, dirección, control; así como, la calidad en salud en las instituciones, constituyen elementos clave para la mejora del desempeño de nuestro sistema de salud. En estos campos destacan los trabajos realizados por un equipo multidisciplinario que incluye a médicos especialistas de Gestión en Salud, cuyos tópicos tratados son: mejora de la calidad de los servicios de salud, satisfacción del usuario, formación de recursos humanos y la relación médico-paciente ${ }^{(22-25)}$.

El uso de evidencias en salud (guías clínicas) y evaluación de tecnologías sanitarias (evaluación con evidencia de medicamentos y dispositivos médicos) son campos importantes donde los médicos especialistas de Gestión en Salud también se encuentran generando información importante para la toma de decisión en salud (UNAGESP - Instituto Nacional de Salud y el IETSI - ESSALUD). Otros campos de desafío en investigación para la especialidad son: la atención domiciliaria, los cuidados paliativos, la telemedicina, entre otros.

\section{CONCLUSIONES}

La especialidad médica actualmente denominada Gestión en Salud, se crea en la Facultad de Medicina de la UNMSM en 1988, como una especialidad para brindar atención integral en el primer nivel de atención de acuerdo a las necesidades prioritarias de salud, pero rápidamente inicia un proceso de conversión que luego de tres fases (inicio, transición y consolidación) ha llegado a concretarse como una especialidad médica que proporciona médicos gestores con conocimientos y habilidades para desarrollar los diversos campos de la gestión en las instituciones del sistema de salud.

Actualmente, la especialización médica de Gestión en Salud de la UNMSM se adquiere mediante el programa de residentado médico, que desarrolla un modelo formativo a través de cursos teóricos y rotaciones de práctica graduales en los tres niveles de gestión (microgestión, mesogestión y macrogestión) del sistema de salud, lo que dota de competencias durante los tres años de formación, donde el egresado culmina el programa siendo capaz de usar 
herramientas y tecnologías que le permiten desempeñarse en los procesos de decisión, conducción, dirección y operatividad de los sistemas de salud del ámbito nacional, regional y local tanto en su componente público como privado.

Contribución de los autores: GFRDR, EV, AEPE participaron en la concepción del artículo; GFRDR, AEPE, NMLC, ICTR, JDCS realizaron la recolección de información; LFRC realizó el procesamiento de datos; LFRC, GFRDR, AEPE, JDCS, JCDCS, NMLC, ICTR realizaron la redacción de artículo; GFRDR, EVV, JCDCS, AEPE, JDCS, LFRC, NMLC, ICTR aprobaron la versión final.

\section{Fuentes de Financiamiento: Autofinanciamiento.}

Conflictos de interés: JCDCS es presidente del Comité Académico de la Especialidad de Gestión en Salud de la UNMSM; GFRDR, AEPE son miembros del comité académico de la especialidad de Gestión en Salud de la UNMSM, JDCS es coordinador académico de la sede docente del Ministerio de Salud de la Especialidad Gestión en Salud.

\section{REFERENCIAS BIBLIOGRÁFICAS}

1. Alemañy E, Díaz-Perera G. Medicina familiar en Cuba. Medwave 2013;13(3):e5634 doi: 10.5867/medwave.2013.03.5634

2. Suárez Rosas L. La especialidad en Organización y Administración de Salud Pública y la Escuela Cubana de Salud Pública. Rev Cuba Salud Pública. 2012;38(3):438-50.

3. Villalbí JR, Pérez-Albarracín G, DelgadoRodríguez M. La formación en España de médicos especialistas en Medicina Preventiva y Salud Pública. Rev Esp Salud Pública. 2011;85(6):507-12.

4. Hospital General Universitario de Elche de España, Unidad Docente de Medicina Preventiva y Salud Pública. Guía docente de formación de residentes de Medicina Preventiva y Salud Pública 2018 [Internet]. Disponible en: http://www.elche.san. gva.es/documents/3462875/3462936/ Guia+docente+MP+2018.pdf

5. Organización Panamericana de la Salud. Residencias en América Latina [Internet]. Washington, D.C: OPS; 2011. 140 p. Disponible en: https://www.paho. org/hq/index.php?option=com_docman\&task $=$ doc_download\&gid $=\overline{1} 6332$ \&Itemid=270\&lang=es

6. Universidad Cayetano Heredia [Internet]. Lima: UPCH; 2019. [citado 3 de marzo de 2019]. Programa de especialidades. Disponible en: https://famed.cayetano.edu. pe/especialidades

7. Universidad Nacional San Agustín [Internet]. Arequipa: UNSA; 2019. [citado 3 de marzo de 2019]. Especialidades. Disponible en: http://fmd.unsa.edu.pe/especialidades/

8. Universidad Privada San Juan Bautista [Internet]. Lima: UPSJB; 2018. [citado 3 de marzo de 2019]. Especialidades. Disponible en: https://residentadomedico. upsjb.edu.pe/especialidades.aspx

9. Universidad Ricardo Palma [Internet]. Lima: URP; 2018. [citado 3 de marzo de 2019]. Especialidades 2018. Disponible en: http://www.urp.edu.pe/administracion/

10. Universidad de la República de Uruguay - Facultad de Medicina. Programa de Formación de Especialistas en Administración de Servicios de Salud. [Internet].
2014. Disponible en: https://higiene.edu. uy/medprev/Prog\%20Adm\%20Serv\%20 Salud\%20'14.pdf

11. Universidad de Chile, Facultad de Medicina [Internet]. Santiago: Uchile; 2019. [citado 3 de marzo de 2019]. Especialista en Salud Pública. Disponible en: http://www. medicina.uchile.cl/postgrados/10001/ salud-publica

12. Universidad de Buenos Aires [Internet]. Buenos Aires: UBA; 2019. [citado 3 de marzo de 2019]. Especialidad de Salud Pública. Disponible en: http://www.uba. ar/posgrados/

13. Gómez L, G. Benavides F. El nuevo programa de la especialidad en medicina preventiva y salud pública: una apuesta por mejorar la práctica profesional. Gac Sanit. 2004;18(2):79-80.

14. Lane DS, Ross V. Defining Competencies and Performance Indicators for Physicians in Medical Management. Am J Prev Med. 1998;14(3):229-36.

15. Henry JB. MD-MBA: A Dual Degree Whose Time Has Come. JAMA. 1987;257(13):1727-8.

16. Ackerly DC, Sangvai DG, Udayakumar K, Shah BR, Kalman NS, Cho AH, et al. Training the next generation of physicianexecutives: an innovative residency pathway in management and leadership. Acad Med J Assoc Am Med Coll. 2011;86(5):575-9.

17. Colegio Médico del Perú [Internet]. Lima: CMP; 2019. [citado 3 de marzo de 2019]. Conoce a tu médico. Disponible en: https://www.cmp.org.pe/conoce-a-tumedico/

18. Mendoza-Arana PJ, Rivera-Del Río G, Gutiérrez-Villafuerte C, SanabriaMontáñez C. El proceso de reforma del sector salud en Perú. Rev Panam Salud Publica. 2018;42:e74. doi: 10.26633/ RPSP.2018.74.

19. Gutierrez EL, Piazza M, GutierrezAguado A, Hijar G, Carmona G, Caballero P, et al. Uso de la evidencia en políticas y programas de salud aportes del Instituto Nacional de Salud. Rev Peru Med Exp Salud Pública. 2016;33(3):5804. doi: 10.17843/rpmesp.2016.333.2308.

20. Ordóñez Crespo CE, Riega López PA, Calisaya Ramírez S. Propuesta metodológica para la planificación de inversiones en infraestructura sanitaria del primer nivel de atención en Essalud. [Tesis de maestría]. Lima: Universidad del Pacifico; 2017 [citado 31 de enero de 2019]. Disponible en: http://repositorio.up.edu. pe/handle/11354/2076

21. Zevallos L, Salas V, Robles L. Asociaciones público-privadas para servicio de salud: ¿la solución al sistema de salud peruano? Rev Peru Med Exp Salud Publica. 2014;31(4):769-74.

22. Miní E, Medina J, Peralta V, Rojas L, Butron J, Gutiérrez EL. Programa de Residentado Médico: Percepciones de los Médicos Residentes en hospitales de Lima y Callao. Rev Peru Med Exp Salud Publica. 2015;32(2):303-10.

23. Podestá Gavilano LE, Maceda Kuljich M. Calidad percibida del servicio y cultura de seguridad en salud en el personal médico del Hospital II EsSalud Vitarte. Lima, 2017. Horiz Méd. 2018;18(3):48-56.

24. Mendoza C, Ramos-Rodríguez C, Gutierrez E. Relación médico-paciente percibida por usuarios de consultorios externos de un hospital de Lima, Perú. Horiz Méd. 2016;16(1):14-9.

25. Gutierrez E, Ramos W, Uribe M, OrtegaLoayza AG, Torres C, Montesinos, et al. Tiempo de espera y su relación con la satisfacción de los usuarios de la farmacia central de un hospital general de Lima. Rev Peru Med Exp Salud Publica. 2009;26(1):61-5.

Correspondencia: Germán Felipe Rivera Del Río

Dirección: Av. Salaverry 1230. Dpto 204 B.

Lima, Perú

Teléfono: (511) 996892245

Correo electrónico: riveradel@hotmail.com 